\title{
Picture Reconstruction from Projections in Restricted Range
}

Alfred Louis

A $79 / 12$

\section{Abstract}

In order to reduce scanning time modern $x$-ray scanners provide projections only in a restricted range $[0, \Phi]$ with $\Phi<\pi$. We consider the reconstruction of pictures from $p+1$ complete projections in $[0, \Phi]$. An extrapolation procedure is given to achieve approximations $g_{p}$ of the data in the whole range. We show that the $\mathrm{L}_{2}$-error of the corresponding picture is of order $\mathrm{p}^{-\alpha}$ if the original belongs to the SOBOLEV space $\mathrm{H}_{0}^{\alpha}$. The validity of our error estimate is investigeted by numerical experiments. 


\section{Introduction}

Scanning with $x$-rays the density $f$ of a tissue lying in a domain $\Omega \subset \mathbb{R}^{2}$ we get the integrals of $f$ along the paths of the rays, see figure 1 ,

(1.1) $g(s, \omega)=\operatorname{Rf}(s, \omega)=\int_{-\infty}^{\infty} f\left(s \omega+t \omega^{\perp}\right) d t$

with $\omega=(\cos \varphi, \sin \varphi), \omega^{\perp}=(-\sin \varphi, \cos \varphi)$. The function $\mathrm{g}=\mathrm{Rf}$ is called the RADON transform of $f$.

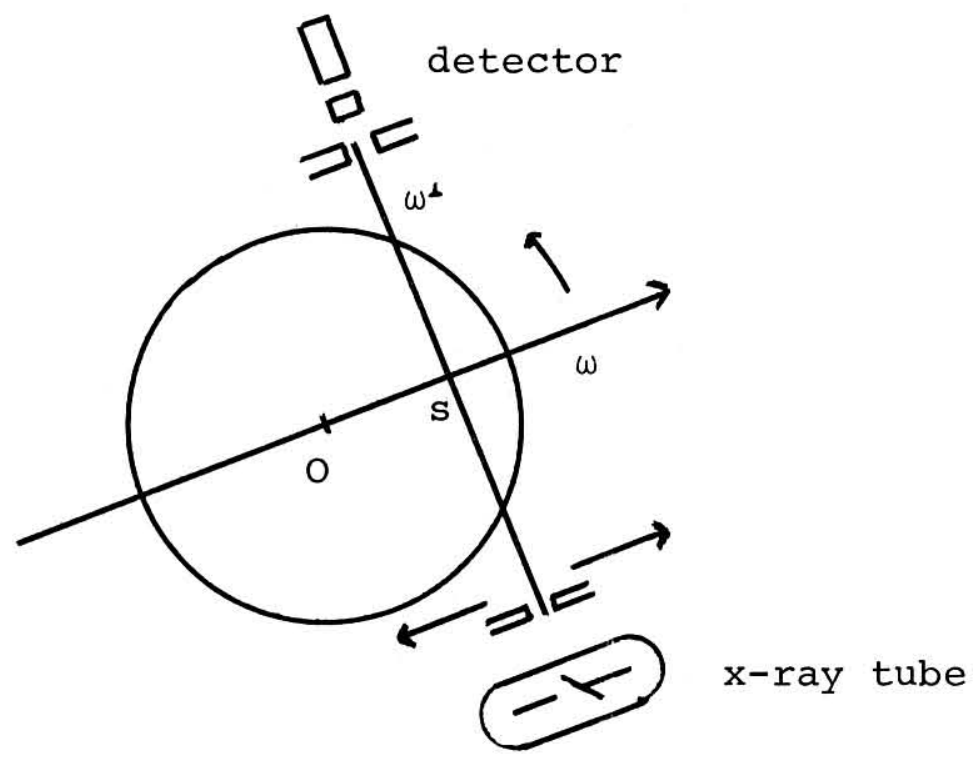

Figure 1.

The reconstruction of the density from its integrals along all lines, i.e. the analytical inversion of the RADON trans form (1.1) requires the values of $\mathrm{g}$ for all $\mathrm{s} \in \mathbb{R}$ and all $\varphi \in[0, \Phi]$ for some $\Phi \in] 0, \pi[$, but the fast reconstruction algorithms need projections in the whole range $[0, \pi[$. In order to apply such a discrete inversion formula projections have to be provided for angles equally distributed over the whole interval $[0, \pi[$. Conventional scanners need several minutes for x-raying, errors occur by motions of the patient, and scanning of the heart for example is impossible. Modern scanners with modified scan geometry need only a few seconds but in general they cannot cover the whole interval $[0, \pi[$. 
The discretization of the integral equation (1.1) and appli cation of ART methods, see GORDON-HERMAN, [2], takes the restricted range into consideration, but the computing time is to large. In order to apply the fast algorithms we have to provide approximations of the data in the missing range. The interpolation of $g$, as proposed by WAGNER, [12], has useful results only if the missing range is sufficiently small.

In $\S 3$ we give an extrapolation algorithm which is applicable without this strong restriction and which achieves better results. The number of arithmetic operations is of the same order as that of the fast reconstruction algorithms and so the procedure is practicable.

In $\S 4$ we show that the approximated data $g_{p}$ lead to a reconstruction $f_{p}$ with $L_{2}$-error of the order $p^{-\alpha}$ if the original picture belongs to the SOBOLEV space $\mathrm{H}_{0}^{\alpha}$. This coincides with the error in using filtered backprojection with $\mathrm{p}$ angles distributed over the whole range as shown in NATTERER, [7].

The numerical examples in $\S 5$ verify the error bounds of $\S 4$ and show that the procedure gives useful results even if the range is only $[0, \Phi]$ with $\Phi=\frac{2}{3} \pi$. 
In this chapter we first introduce the SOBOLEV spaces used in the following. Let $\mathrm{k}$ be an integer and $\mathrm{G} \subset \operatorname{IR}^{\mathrm{k}}$ be an open and bounded domain. $C_{O}^{\infty}(G)$ is the set of all real-valued infinitely differentiable functions with compact support in $G$. For real $\alpha$ we denote by $\mathrm{H}^{\alpha}\left(\mathbb{I R}^{\mathrm{k}}\right)$ the SOBOLEV space of order $\alpha$, i.e. the set of all tempered distributions with

$$
\text { (2.1) } \quad\|f\|_{H^{\alpha}\left(\mathbb{R}^{k}\right)}^{2}=\int_{\mathbb{R}^{k}}\left(1+|\xi|^{2}\right)^{\alpha}|\hat{f}(\xi)|^{2} \mathrm{~d} \xi<\infty
$$

where $\hat{f}$ is the FOURIER transform of $f$ :

$$
\text { (2.2) } \hat{f}(\xi)=\int f(x) e^{-2 \pi i} x \cdot \xi d x .
$$

For domains $\mathrm{G}$ with sufficiently regular boundary we put

(2.3) $H_{O}^{\alpha}(G)=\left\{f \in H^{\alpha}\left(I^{k}\right): \operatorname{supp}(f) \subset \bar{G}\right\}$,

(2.4) $\|f\|_{H_{O}^{\alpha}(G)}=\|f\|_{H^{\alpha}\left(I^{k}\right)}$ for $f \in H_{O}^{\alpha}(G)$.

These spaces are the $\tilde{\mathrm{H}}_{2}^{\alpha}(\mathrm{G})$ spaces of TRIEBEL, [11], chapt.

4.3.2 and for $\alpha \neq k+1 / 2, k$ integer, they coincide with the closure of $\mathrm{C}_{\mathrm{O}}^{\infty}(\mathrm{G})$ in the $\mathrm{H}^{\alpha}$ - norm. For all $\alpha, \beta \in \mathbb{R}$ and $0 \leqq \theta \leqq 1$ they fulfill the interpolation property

(2.5) $\quad\left[\mathrm{H}_{\mathrm{O}}^{\alpha}(\mathrm{G}), \mathrm{H}_{\mathrm{O}}^{\beta}(\mathrm{G})\right]_{\theta}=\mathrm{H}_{\mathrm{O}}^{\alpha(1-\theta)+\beta \theta}(\mathrm{G})$

where $[\mathrm{X}, \mathrm{Y}]_{\theta}$ denotes the interpolation space of definition 2.1 in LIONS-MAGENES, [4].

For a real interval $I$ and a weight function $\mathrm{p}$ we define the SOBOLEV space with weight

$$
\mathrm{W}_{2}^{\mathrm{S}}(\mathrm{I}, \mathrm{p}, 1), \mathrm{s} \geqq 0,
$$

as the set of all $\mathrm{L}_{2}$ functions with $\|f\|_{W_{2}^{S}(I, p, 1)}<\infty$

If $\mathrm{s}$ is an integer this norm is defined as

(2.6) $\|f\|_{W_{2}^{S}(I, p, 1)}=\left[\int_{I}\left(p(x)\left|f^{(s)}(x)\right|^{2}+|f(x)|^{2}\right) d x\right]^{1 / 2}$ and for $\mathrm{s}=\mathrm{k}+\sigma, \mathrm{k} \in \mathrm{IN}_{\mathrm{O}}$ one sets 
(2.7) $\|f\|_{W_{2}^{S}(I, p, 1)}=\left[\int_{I} \int_{I} \frac{\left|p^{1 / 2}(x) f^{(k)}(x)-p^{1 / 2}(y) f^{(k)}(y)\right|^{2}}{|x-y|^{1+2 \sigma}} d x d y\right]^{1 /}$

$$
+\|f\|_{W_{2}^{k}(I, p, 1)} \text {, }
$$

see TRIEBEL, [10], chapt. 3.2.6.

Finally let $S$ be the boundary of the unit circle in $\operatorname{IR}^{2}$ and $\mathrm{Z}=\mathbb{R} \times \mathrm{S} \subset \mathbb{I R}^{3}$. On the manifold $\mathrm{Z}$ we use the SOBOLEV space $\mathrm{H}^{\alpha}(\mathrm{z})$ with the norm

(2.8) $\quad\|g\|_{H^{\alpha}(Z)}=\left[\int_{0}^{2 \pi}\|g(\cdot, \omega)\|_{H^{\alpha}(I R)}^{2} d \varphi\right]^{1 / 2}$

for $\alpha \geqq 0$.

Next we consider the RADON transform in the SOBOLEV space $\mathrm{H}_{\mathrm{O}}^{\alpha}(\Omega)$ where $\Omega \subset \mathbb{I R}^{2}$ is bounded. The line integrals in (1.1) are defined for $f \in \mathrm{H}_{\mathrm{O}}^{\alpha}(\Omega)$ for $\alpha>1 / 2$. For $f \in \mathrm{C}_{\mathrm{O}}^{\infty}(\Omega)$ the norms $\|f\|_{\mathrm{H}_{\mathrm{O}}^{-1 / 2}(\Omega)}$ and $\|\mathrm{Rf}\|_{\mathrm{L}_{2}(\mathrm{Z})}$ are equivalent, see NATTERER, [7],

theorem 3.1. So we can extend the RADON transform to all of $\mathrm{H}_{0}^{-1 / 2}(\Omega)$ by continuity because $\mathrm{C}_{0}^{\infty}(\Omega)$ is dense in $\mathrm{H}_{0}^{\alpha}(\Omega)$; the extension is again denoted by $R$.

\section{THEOREM 2.1}

For real $\alpha \geqq-\frac{1}{2}$ the RADON transform is a bounded operator from $\mathrm{H}_{\mathrm{O}}^{\alpha}(\Omega)$ into $\mathrm{H}^{\alpha+1 / 2}(\mathrm{z})$.

PROOF

For $\alpha>1 / 2$ this is theorem 3.2 in NATTERER, [7]. Theorem 3.1 in [7] states that $\|R f\|_{L_{2}(Z)} \leqq c\|f\|_{H_{O}^{-1 / 2}(\Omega)}$ for $f \in C_{O}^{\infty}(\Omega)$.

This is also true for the extension of $R$ on $\mathrm{H}_{0}^{-1 / 2}(\Omega)$. Now the result follows from the interpolation theorem 5.1, chapt. 1 of LIONS-MAGENES, [4]. 
The extrapolation procedure given here is based upon the PALEY-WIENER theorem for the RADON transform. It characterizes the small range of that ill-posed problem.

\section{THEOREM 3.1}

A function $g \in \mathrm{H}^{1 / 2}(\mathrm{z})$ with compact support is the RADON trans form of a function $f \in L_{2}\left(\mathbb{R}^{2}\right)$ with compact support if and only if

$$
\text { i) } g(-s,-\omega)=g(s, \omega) \text { on } z \text {, }
$$

ii) for all $\mathrm{m} \in \mathbb{I N}_{\mathrm{O}}$

$$
\int_{\mathbb{R}} s^{m} g(s, \omega) d s
$$

is a homogeneous polynomial of degree $\leqq m$ in $\omega$.

For a proof see LAX-PHILLIPS, [3]. This result suggests a series expansion of $\mathrm{g}$ in terms of polynomials, and especially the LEGENDRE polynomials are well suited.

Let us assume that the tissue to be scanned is lying in a circle $\Omega$ around $O$ with radius $1 / 2$. That means that the support of the density $f$ is lying in $\Omega$ and the support of the functions $g(\cdot, \omega)$ is in $[-1 / 2,1 / 2]$ for all $\varphi$. Further we assume that we have $p+1$ complete projections in the interval $[0, \Phi]$ with $\Phi<\pi$, i.e. the functions

$$
g\left(\cdot, \omega_{j}\right), j=0, \ldots, p
$$

with $\omega_{j}=\left(\cos \varphi_{j}, \sin \varphi_{j}\right), \varphi_{j} \in[0, \Phi], \varphi_{j} \neq \varphi_{k}$ for $j \neq k$

are known. In practice this means that at sufficiently many positions $s$ for each $\omega_{j}$ the tissue is scanned.

Let us denote with $Q_{\mathrm{m}}$ the normalized LEGENDRE polynomials of degree m. From theorem 3.1 we know that the coefficients of the expansion in terms of LEGENDRE polynomials

$$
q_{m}(\varphi)=\int_{-1}^{1} Q_{m}(s) g(s, \omega) d s
$$

are polynomials of degree less or equal $\mathrm{m}$ in $\omega$. Because of 
the periodicity of $g$ the $\mathrm{q}_{\mathrm{m}}$ have the representation

$$
q_{m}(\varphi)=\sum_{k=0}^{[m / 2]}\left(a_{k}^{m} \cos c_{k}^{m}+b_{k}^{m} \sin c_{k}^{m}\right),
$$

where $\left[\mathrm{x}\right.$ ] denotes the integral part of $\mathrm{x}$ and the $\mathrm{a}_{\mathrm{k}}^{\mathrm{m}}, \mathrm{b}_{\mathrm{k}}^{\mathrm{m}}$ are coefficients and

$$
c_{k}^{m}= \begin{cases}2 k & \text { for } m \text { even } \\ 2 k+1 & \text { for } m \text { odd }\end{cases}
$$

The $\mathrm{q}_{\mathrm{m}}$ defined in (3.3) can be calculated for the angles $\varphi_{j}, j=0, \ldots, p$ and then $(3.4)$ is a system of linear equations for the coefficients $a_{k}^{m}, b_{k}^{m}$. This system is overdetermined for $\mathrm{m} \leqq \mathrm{p}$ and can be solved for example by singular value decom position. Now for those $m$ the coefficients $q_{m}$ of the series expansion are detemined and we have the following approxi mation of the function $g$ :

$$
g_{p}(s, \omega)=\left\{\begin{array}{cl}
\sum_{m=0}^{p} Q_{m}(s) q_{m}(\varphi) & \text { for }|s|<1 \\
0 & \text { for }|s| \geqq 1
\end{array}\right.
$$

With this function, approximations for the data in the missing range can be calculated. If the angles in $[0, \Phi]$ are not equally distributed it is possible to compute the data for equally distributed angles in the whole range. With $\theta$ we denote

$$
\theta=[\pi / \Phi] \text {. }
$$

This leads to the following algorithm

STEP 1 : Compute $q_{m}\left(\varphi_{j}\right)$ according to $(3.3)$ for $j=0, \ldots, p$, $\mathrm{m}=0, \ldots, \mathrm{p}$.

STEP 2 : Solve the system of equations (3.4) with $\varphi=\varphi_{j}$ for $j, m=0, \ldots, p$.

STEP 3 : Compute $q_{m}\left(\varphi_{j}\right)$ according to $(3.4)$ for $m=0, \ldots, p$ and $j=p+1, \ldots, \theta p-1$, or $q_{m}\left(\psi_{j}\right)$ for $m=0, \ldots, p$ and $j=0, \ldots, \theta p-1$

with $\psi_{j}$ equally distributed over $[0, \pi[$.

STEP 4 : Compute $g_{p}\left(s_{i}, w_{j}\right), j=p+1, \ldots, \theta p-1$ or

$$
\text { for } i=-q, \ldots, q-1 \text {. }
$$

$$
\begin{aligned}
& j=0, \ldots, \theta p-1, \\
& \sim_{j}=\left(\cos \psi_{j}, \sin \psi_{j}\right)
\end{aligned}
$$


The count of operations shows that we have a procedure with $O\left(p^{s}{ }^{t}\right)$ arithmetic operations where $s+t=3$. This is the same order as for the fast reconstruction algorithms. If we calculate the integrals in step 1 with the trapezoidal rule at the points $s_{i}, i=-q, \ldots, q-1$, we need $2 q(p+1)^{2}$ operations. The singular value decomposition in step 2 takes more effort, but for each scanner the directions $\omega_{j}$ are known a priori, so the matrices in (3.4) can be calculated and be decomposed independently of the data once and for all. In the actual computation we then have to compute only products of matrices and vectors, which requires $\mathrm{cp}^{3}$ operations. In step 3 there are at most $\frac{1}{2} \theta p(p+1)^{2}$ operations and finally $2 \theta p^{2} q$ operations in step 4 . 


\section{4}

Error Estimates

In this chapter we prove error estimates in SOBOLEV norms for the picture reconstructed from the data $g_{p}$ instead of $g$. First we show that $g_{p}$ lies in the range of the RADON transform.

\section{LEMMA 4.1}

Let $g_{p}$ be defined as in (3.6).

Then there exists $f_{p} \in L_{2}\left(\mathbb{R}^{2}\right)$ with compact support and

$$
g_{p}(s, \omega)=\int f_{p}\left(s \omega+t \omega^{1}\right) d t
$$

PROOF

The function $g_{p}$ is in $C^{\infty}([-1,1] \times s)$ and has compact support. So we can use theorem 3.1 to prove this lemma. First we show the symmetry of $g_{p}$

$$
g_{p}(-s,-\omega)=g_{p}(s, \omega) \text { on } z \text {. }
$$

Because of $-\omega=(-\cos \varphi,-\sin \varphi)=(\cos (\varphi+\pi), \sin (\varphi+\pi))$ we get with the addition formula for sin and cos that $\cos c_{k}^{m}(\varphi+\pi)=(-1)^{m} \cos c_{k}^{m}$ and $\sin c_{k}^{m}(\varphi+\pi)=(-1)^{m} \sin c_{k}^{m}$ and so

$$
\mathrm{q}_{\mathrm{m}}(\varphi+\pi)=(-1)^{\mathrm{m}} \mathrm{q}_{\mathrm{m}}(\varphi) \text {. }
$$

Now (4.2) follows from the symmetry of the LEGENDRE polynomials, i.e. $Q_{m}(-s)=(-1)^{m} Q_{m}(s)$.

The second condition in theorem 3.1 for $g_{p}$ is a consequence of the orthogonality of the LEGENDRE polynomials. Each monomial $\mathrm{s}^{\mathrm{m}}$ can be represented in the form

$$
s^{m}=\sum_{j=0}^{m} d_{j}^{m} Q_{j}(s) \text { with } d_{j}^{m}=0 \text { for } j+m \text { odd. }
$$

So we get

$$
\begin{aligned}
\int s^{m} g_{p}(s, \omega) d s & =\sum_{k=0}^{p} q_{k}(\varphi) \int s^{m} Q_{k}(s) d s \\
& =\sum_{k=0}^{p} q_{k}(\varphi) \sum_{j=0}^{m} d_{j}^{m} \int Q_{j}(s) Q_{k}(s) d s \\
& =\sum_{k=0}^{t} q_{k}(\varphi) d_{k}^{m}
\end{aligned}
$$

where $t=\min (p, k)$. 
The $q_{m}$ defined in (3.3) can be represented by a homogeneous polynomial of degree $\leqq m$ because of (3.4), (3.5), which completes the proof.

Next we give an error estimate for the partial sum of an expansion of a function in terms of LEGENDRE polynomials. Let $I$ be the interval ]-1,1[ and $p$ be the weight function $\mathrm{p}(\mathrm{x})=1-\mathrm{x}^{2}$. With $\mathrm{K}^{\mathrm{S}}$ we denote

$$
\mathrm{K}^{\mathrm{S}}=\mathrm{W}_{2}^{\mathrm{S}}\left(\mathrm{I}, \mathrm{p}^{\mathrm{S}}, 1\right) \text { for } \mathrm{s} \neq \frac{1}{2}+\mathrm{k} \text {, where } \mathrm{k} \in \mathbf{N}_{\mathrm{O}}
$$

and for $s=\frac{1}{2}+k, k \in \mathbb{N}_{\mathrm{O}}$, the space $\mathrm{K}^{\mathrm{S}}$ is the completion of $\mathrm{C}^{\infty}(\overline{\mathrm{I}})$ in the norm

$$
\text { (4.3) } \quad\|\mathrm{u}\|_{\mathrm{K}^{\mathrm{S}}}=\left[\|\mathrm{u}\|_{\mathrm{W}_{2}^{\mathrm{S}}\left(\mathrm{I}, \mathrm{p}^{\mathrm{s}}, 1\right)}^{2}+\int_{-1}^{1} \mathrm{p}^{\mathrm{s}-1}(\mathrm{x})\left|\mathrm{u}^{(\mathrm{k})}(\mathrm{x})\right|^{2} \mathrm{dx}\right]^{1 / 2} \text {. }
$$

\section{LEMMA 4.2}

Let $\mathrm{u} \in \mathrm{K}^{t}$ and $\mathrm{u}_{\mathrm{p}}$ be the partial sum of the expansion of $\mathrm{u}$ in terms of LEGENDRE polynomials

$$
u_{p}(x)=\sum_{k=0}^{p} c_{k} Q_{k}(x)
$$

with

(4.4) $\quad c_{k}=\int_{-1}^{1} u(x) Q_{k}(x) d x$.

Then there is a constant $\mathrm{c}>\mathrm{O}$ independent of $\mathrm{u}$ and $\mathrm{p}$ such that

$$
\text { (4.5) } \quad\left\|u-u_{p}\right\|_{K^{s}} \leqq c p^{-(t-s)}\|u\|_{K}{ }^{t}
$$

for $0 \leqq s \leqq t$.

COROLLARY 4.3

Let $\gamma \in] 0,1\left[\right.$ and $u \in H_{O}^{t}(]-\gamma, \gamma[)$,

then

(4.6) $\quad\left\|u-u_{p}\right\|_{H^{S}(]-\gamma, \gamma[)} \leqq c_{1} p^{-(t-s)}\|u\|_{H} t_{(]-\gamma, \gamma[)}$

for $0 \leqq s \leqq t$. 


\section{PROOF}

Let $A$ be the LEGENDRE differential operator $-\frac{d}{d x}\left(\left(1-x^{2}\right) \frac{d}{d x}\right)$ and $\bar{A}$ be the closure of $A$. Then it is shown in TRIEBEL, [11], chapt. 7.7 .1 that

$$
D\left(\bar{A}^{S}\right)=K^{2 s} \text { if } s \geqq 0 \text {. }
$$

$\overline{\mathrm{A}}^{\mathrm{S}}$ is a selfadjoint operator with the eigenfunctions $\mathrm{Q}_{\mathrm{k}}$ and the eigenvalues $\lambda_{\mathrm{k}}^{\mathrm{s}}$ with

$$
\lambda_{\mathrm{k}}=\mathrm{k}(\mathrm{k}+1) \text {. }
$$

Following LIONS-MAGENES, [4], chapt. 1, formula (16.12) we define the norm

(4.7) $\quad\|\mathrm{u}\|_{\mathrm{K}^{\mathrm{s}}}^{\mathrm{s}}=(\mathrm{u}, \overline{\mathrm{A}} \mathrm{su})_{\mathrm{L}_{2}}^{1 / 2}=\left[\sum_{\mathrm{k}=0}^{\infty} \lambda_{\mathrm{k}}^{\mathrm{s}} \mathrm{c}_{\mathrm{k}}^{2}\right]^{1 / 2}$

with $\mathrm{c}_{\mathrm{k}}$ as defined in (4.4). This norm is equivalent with the $\mathrm{K}^{\mathrm{S}}$ norm defined above. Then we have

$$
\begin{aligned}
\left\|u-u_{p}\right\|_{K^{s}}^{2} & \leqq c^{\prime}\left\|u-u_{p}\right\| k_{K^{s}}^{2} \\
& =c^{\prime} \sum_{k=p+1}^{\infty}[k(k+1)]^{s} c_{k}^{2} \\
& =c^{\prime} \sum_{k=p+1}^{\infty}[k(k+1)]^{s-t}[k(k+1)]^{t} c_{k}^{2} \\
& \leqq c^{\prime}[(p+1)(p+2)]^{s-t} \sum_{k=p+1}^{\infty}[k(k+1)]^{t} c_{k}^{2} \\
& \leqq c^{\prime}{ }^{\prime}[(p+1)(p+2)]^{s-t}\|u\|_{k}^{t}
\end{aligned}
$$

which completes the proof of lemma 4.2. Now the result of the corollary follows from the fact that the weight function $p$ is bounded away from zero on the interval $[-\gamma, \gamma]$ for $0<\gamma<1$. So the norms $\|\cdot\|_{K^{S}}$ and $\|\cdot\|_{H^{S}(]-\gamma, \gamma[)}$ are equivalent for functions with support in $[-\gamma, \gamma]$. 
Now we are able to give an error estimate for the function $f_{p}$ defined in (4.1).

\section{THEOREM 4.4}

Let $\mathrm{f} \in \mathrm{H}_{\mathrm{O}}^{\alpha}(\Omega), \alpha>0$, and $\mathrm{f}_{\mathrm{p}}$ be defined as in (4.1).

Then we have

(4.10) $\quad\left\|f-f_{p}\right\|_{H_{O}^{\beta}(\Omega)} \leqq c p^{-(\alpha-\beta)}\|f\|_{H_{O}^{\alpha}(\Omega)}$

for $-\frac{1}{2} \leqq \beta<\alpha$.

PROOF

The proof is based upon the continuity of $\mathrm{R}^{-1}$ from a subspace of $\mathrm{H}^{\alpha+1 / 2}(\mathrm{z})$ into $\mathrm{H}_{\mathrm{O}}^{\alpha}(\Omega)$, see SMITH-SOLMON-WAGNER, [10], theorem 12.6 .

For $\beta \geqq-\frac{1}{2}$ we have

$$
\begin{aligned}
\left\|f-f_{p}\right\|_{H_{0}^{\beta}(\Omega)}^{2} & \leqq c\left\|g-g_{p}\right\|_{H^{\beta+1 / 2}(z)}^{2} \\
& =c \int_{0}^{2 \pi}\left\|\left(g-g_{p}\right)(\cdot, \omega)\right\|_{H^{\beta+1 / 2}(I)}^{2} d \varphi \\
& \leqq \bar{c} p^{-2(\alpha-\beta)} \int_{0}^{2 \pi}\|g(\cdot, \omega)\|_{H^{\alpha+1 / 2}(I)}^{2} d \varphi \\
& =\bar{c} p^{-2(\alpha-\beta)}\|g\|_{H^{\alpha+1 / 2}(Z)}^{2} \\
& \leqq \bar{c} p^{-2(\alpha-\beta)}\|f\|_{H_{0}^{2}(\Omega)}^{2}
\end{aligned}
$$

where we have used corollary 4.3 with $I=]-\frac{1}{2}, \frac{1}{2}[$ and the regularity theorem 2.1 . 


\section{REMARKS}

1. In the important case $\mathrm{f} \in \mathrm{H}_{\mathrm{O}}^{1 / 2}(\Omega)$ we have a $\mathrm{L}_{2}$ error of order $\mathrm{p}^{-1 / 2}$.

2. If one uses the filtered backprojection algorithm as described in RAMACHANDRAN-LAKSHMINARAYANAN, [9], with $\mathrm{p}_{1}=\theta \mathrm{p}, \theta \sim \pi / \Phi$, directions with the ideal low pass filter and cut off frequency $\sigma_{0}=\mathrm{cp}$ and the approximated data $\mathrm{g}_{\mathrm{p}_{-\alpha}}$, then the total error in $\mathrm{L}_{2}$ is also of the order $\mathrm{p}^{-\alpha}$, see NATTERER, [7]. 
In order to investigate the error estimate of theorem 4.4 we consider first a rather simple example.

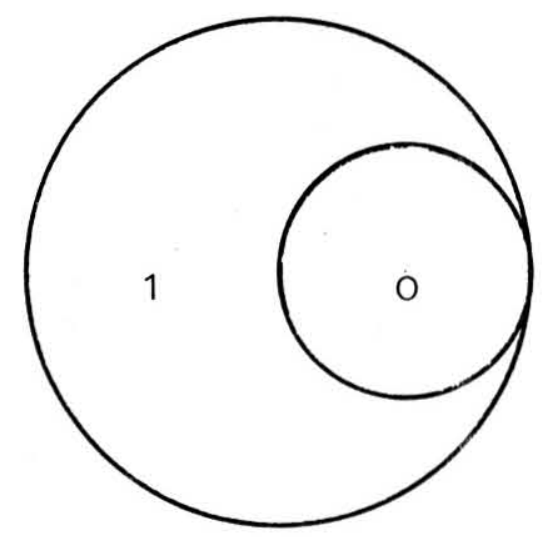

Figure 2. First test object

The function $\mathrm{f} \in \mathrm{H}_{\mathrm{O}}^{\mathrm{S}}(\Omega), \mathbf{s}<1 / 2$, as shown in figure 2 is defined as 1 in $\left\{\mathrm{x} \in \mathbb{R}^{2}:|\mathrm{x}|<0.3 \wedge|\mathrm{x}-(0.15,0)|>0.15\right\}$ and $O$ otherwise. To simulate the fact of complete projections we have analytically computed the function $g\left(s, \omega_{j}\right)$ with a stepsize $1 / 256$ in the $s$ - direction and for $p+1$ angles in the range $[0,2 / 3 \pi]$, i.e. within a range of $120^{\circ}$. To reconstruct $f$, we have used the filtered backprojection with $3 p / 2$ angles equally distributed over $[0, \pi[$. The results are given in table 1 and 2 and show the order $\mathrm{p}^{-1 / 2}$ for the $\mathrm{L}_{2}$-error. The function $f_{p}^{1}$ is reconstructed using $g_{p}\left(s, \omega_{j}\right)$ for all $j=0, \ldots, \frac{3}{2} p-1$, see table 1 , in the second case the data are approximated only in the interval $] \frac{2}{3} \pi, \pi\left[\right.$ and in $\left[0, \frac{2}{3} \pi\right]$ the given exact data are used, see table 2. Finally table 3 shows the result when only exact data are used. 
Table 1
Projections

$$
\left\|f-f_{p}^{1}\right\|_{L_{2}}
$$$$
\mathrm{p}^{1 / 2}\left\|\mathrm{f}-\mathrm{f}_{\mathrm{p}}^{1}\right\|_{\mathrm{L}_{2}}
$$
20$$
1.8026 \cdot 10^{-1}
$$
0.81
30
$1.5635 \cdot 10^{-1}$
0.86
40
$1.3255 \cdot 10^{-1}$
0.84
60
$1.0842 \cdot 10^{-1}$
0.84

$\mathrm{L}_{2}$-error of the reproduction $\mathrm{f}_{\mathrm{p}}^{1}$ if all the data are approximated by $g_{p}$.

Table 2

Projections

$$
\left\|f-f_{p}^{2}\right\|_{L_{2}}
$$$$
\mathrm{p}^{1 / 2}\left\|\mathrm{f}-\mathrm{f}_{\mathrm{p}}^{2}\right\|_{\mathrm{L}_{2}}
$$

20

$1.1378 \cdot 10^{-1}$

30

$1.0031 \cdot 10^{-1}$

0.51

40

$8.4308 \cdot 10^{-2}$

0.55

$6.8286 \cdot 10^{-2}$

0.53

60

$$
6.8286 \cdot 10^{-2}
$$$$
0.53
$$

$\mathrm{L}_{2}$-error of the reproduction $\mathrm{f}_{\mathrm{p}}^{2}$ if the data are approximated by $\mathrm{g}_{\mathrm{p}}$ only in the missing range.

Table 3

$\begin{array}{llc}\text { Projections } & \left\|f-f_{p}^{3}\right\| L_{2} & p^{1 / 2}\left\|f-f_{p}^{3}\right\| L_{2} \\ 20 & 8.9923 \cdot 10^{-2} & 0.40 \\ 30 & 7.0715 \cdot 10^{-2} & 0.39 \\ 40 & 5.9519 \cdot 10^{-2} & 0.38 \\ 60 & 4.7400 \cdot 10^{-2} & 0.37\end{array}$

$\mathrm{L}_{2}$-error of the reproduction $\mathrm{f}_{\mathrm{p}}^{3}$ if the data are exact. 
Next we investigated our procedure in a more realistic case. We considered a second test object simulating the case of a tumor in the brain.

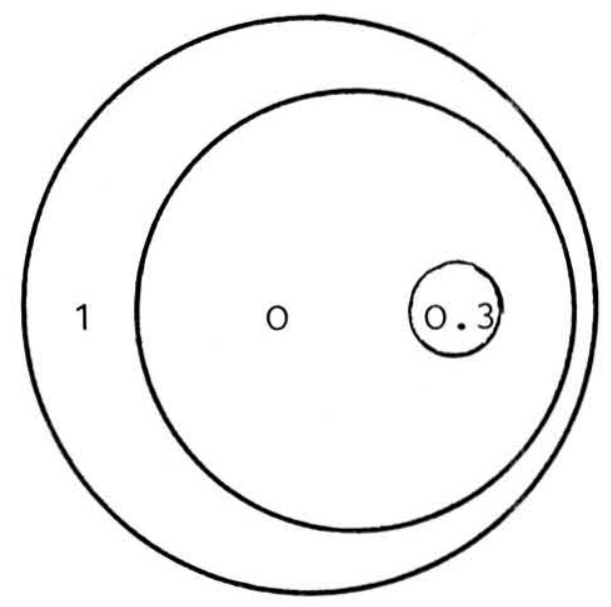

Figure 3. Second test object

The function $f$ is again in $\mathrm{H}_{0}^{\mathrm{S}}(\Omega), \mathrm{s}<1 / 2$, and defined as 1 in $\left\{x \in \mathbb{R}^{2}:|x|<0.3 \wedge|x-(0.05,0)|>0.225\right\}$, as 0.3 in $\left\{\mathrm{x} \in \mathbb{R}^{2}:|\mathrm{x}-(0.15,0)|<0.05\right\}$ and 0 otherwise.

Here the integrals in (3.3) have to be computed numerically. We used the trapezoidal rule with stepsize $\frac{1}{256}$. The range where the data are given is again $\left[0, \frac{2}{3} \pi\right]$. Here we used $\mathrm{p}=30,40,60$ and equidistributed angles. The picture is reconstructed with the filtered backprojection and $\frac{3}{2} p$ equidistributed angles. These results are compared with that obtained from $\mathrm{p}+1$ projections in $\left[0, \pi\left[\right.\right.$. The $\mathrm{L}_{2}$-errors are given in table 4 and $3-D$ pictures over a $64 \times 64$ grid in the figures 4-8. Figure 4 shows the original function $\mathrm{f}$, figures 5,6 the reconstructed function from 40 projections and figure 7,8 from 60 projections in $\left[0, \frac{2}{3} \pi\right]$ resp. $[0, \pi[$. Finally figure 9 shows a cut of these five functions at $\mathrm{y}=0$. 
Table 4
Projections
$\left\|f-f_{p}^{1}\right\|_{L_{2}}$
$\left\|f-f_{p}^{2}\right\|_{L_{2}}$
30
$1.2651 \cdot 10^{-1}$
$9.3582 \cdot 10^{-2}$
40
$1.0433 \cdot 10^{-1}$
$7.8882 \cdot 10^{-2}$
60
$9.3725 \cdot 10^{-2}$
$6.9419 \cdot 10^{-2}$

$\mathrm{L}_{2}$-errors of the reproduction of test-object 2 from $\mathrm{p}$ projections in $\left[0, \frac{2}{3} \pi\right]$ and $[0, \pi[\mathrm{resp}$. 


\section{Figure 4}

3-D picture of the second test object, see figure 3

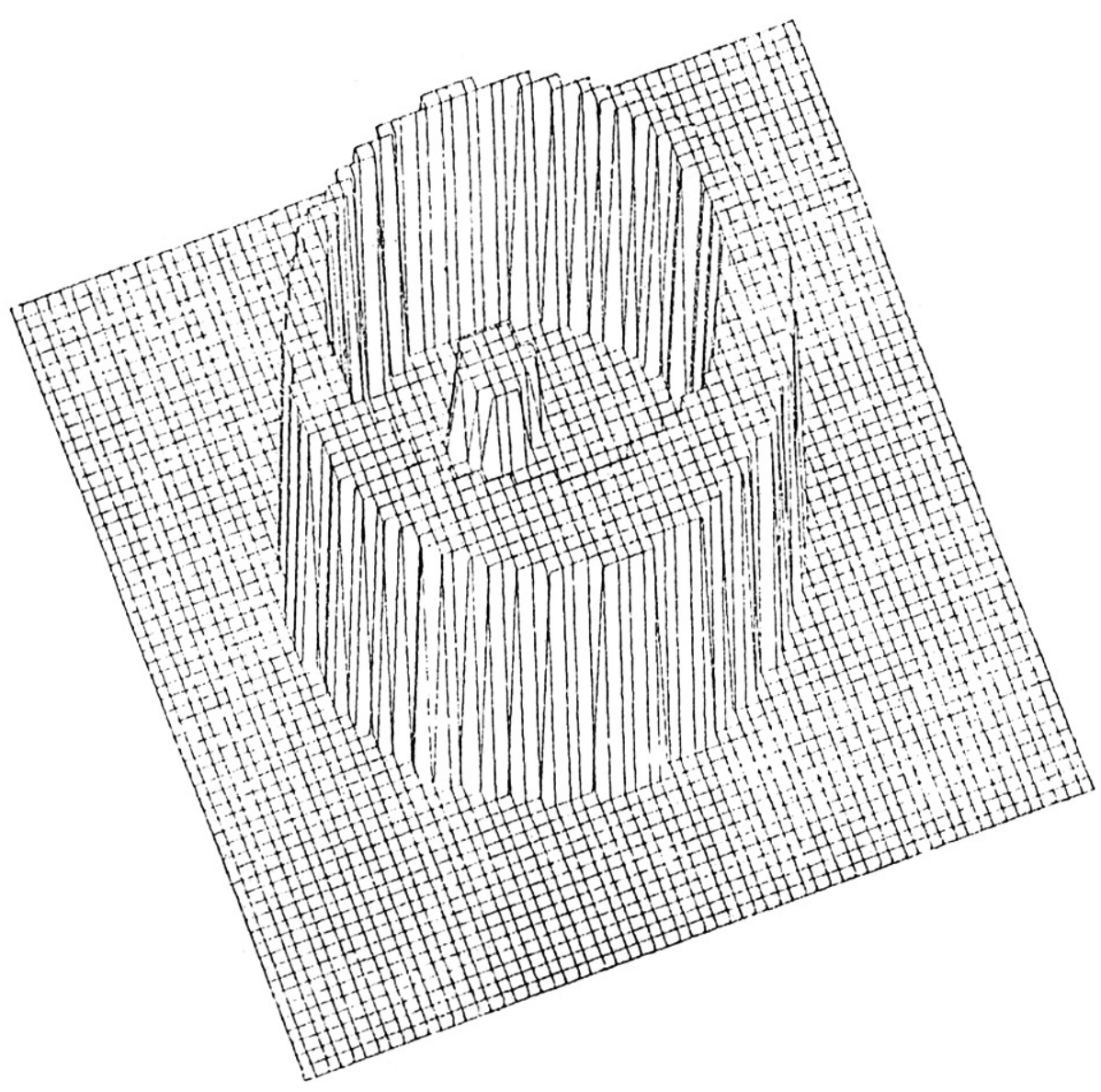




\section{Figure 5}

Reconstruction of the second test object from 40 projections in $\left[0, \frac{2}{3} \pi\right]$

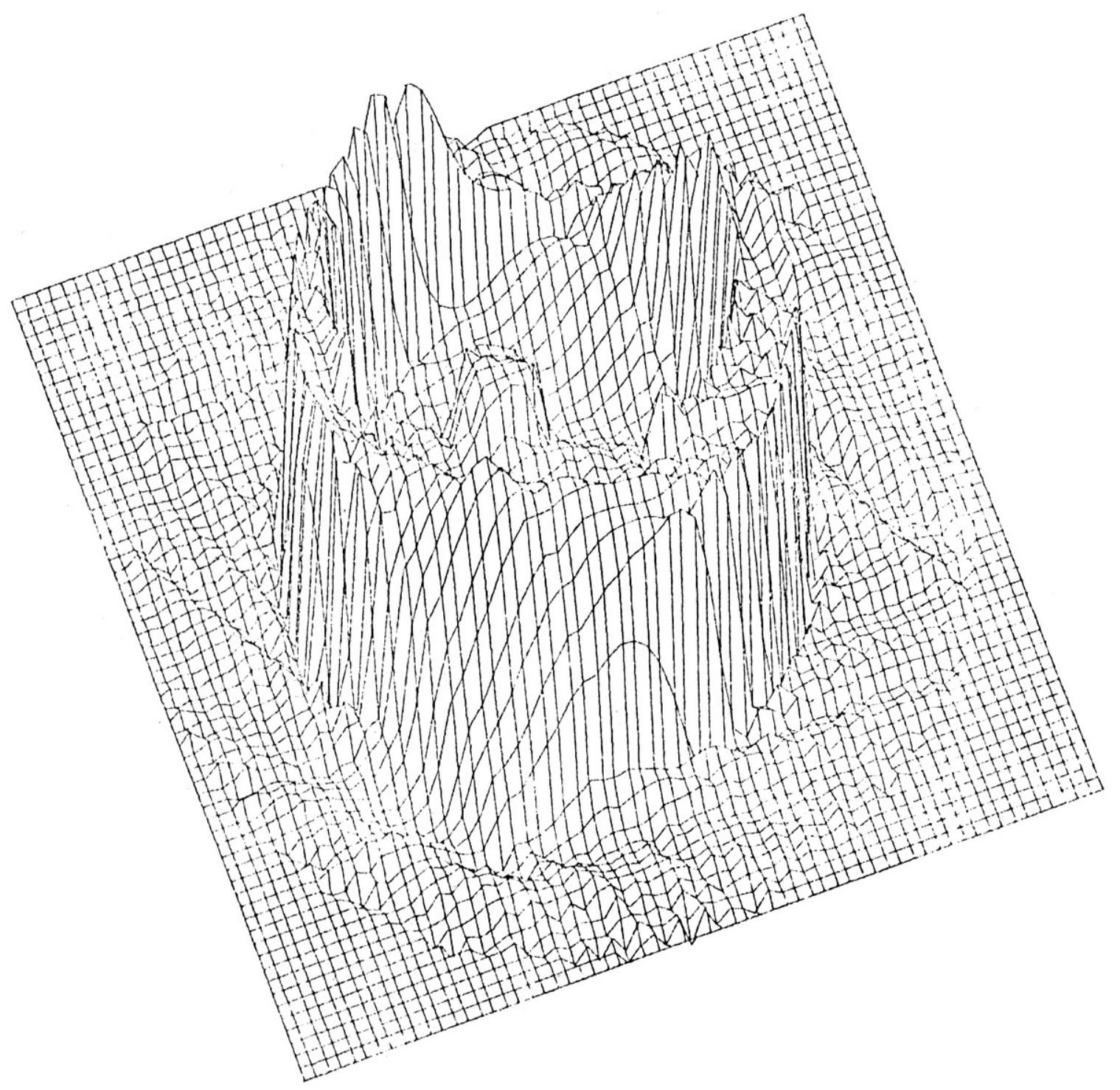


Figure 6

Reconstruction of the second test object from 40 projections in $[0, \pi[$

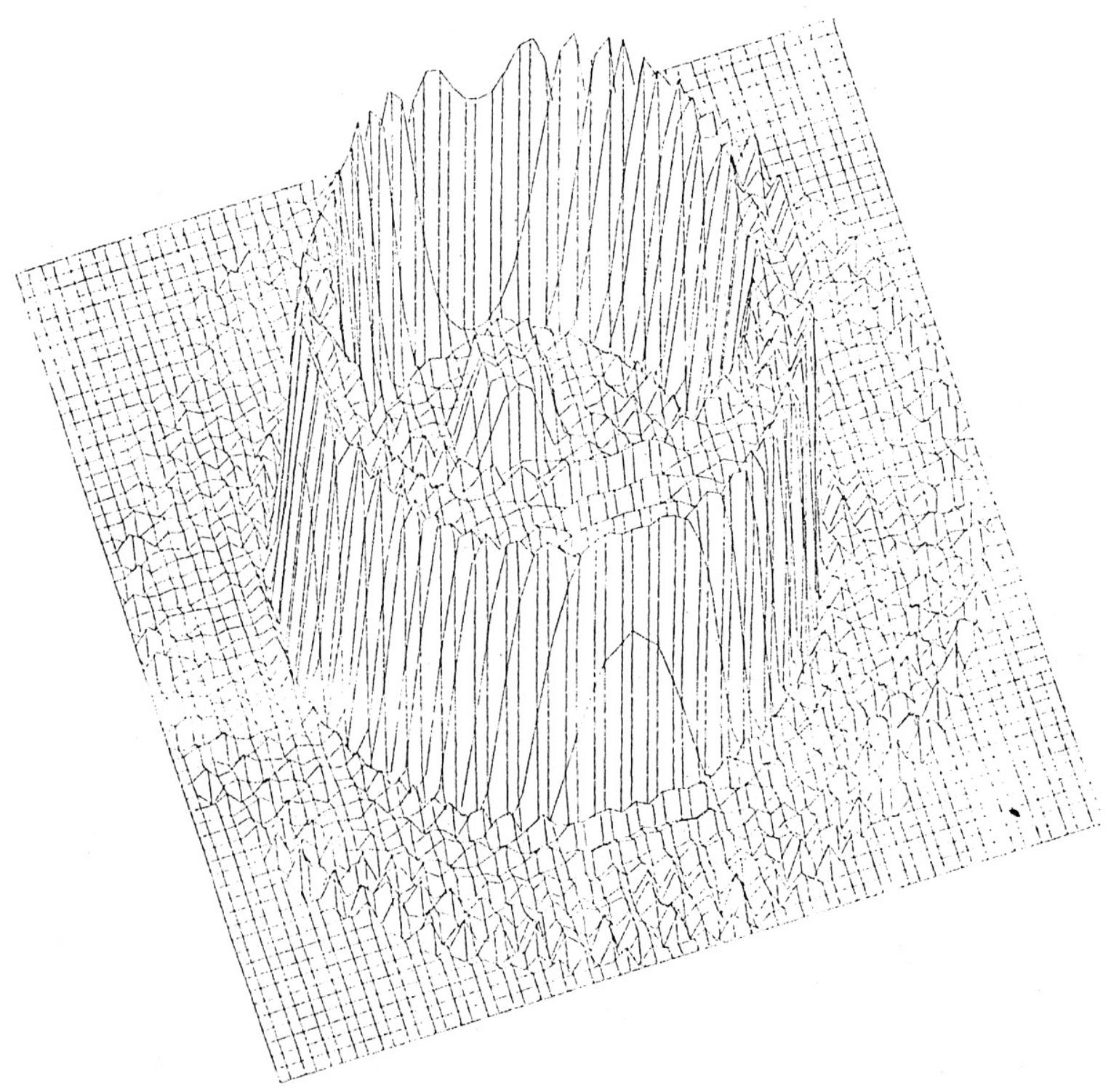


Figure 7

Reconstruction of the second test object from 60 projections in $\left[0, \frac{2}{3} \pi\right]$

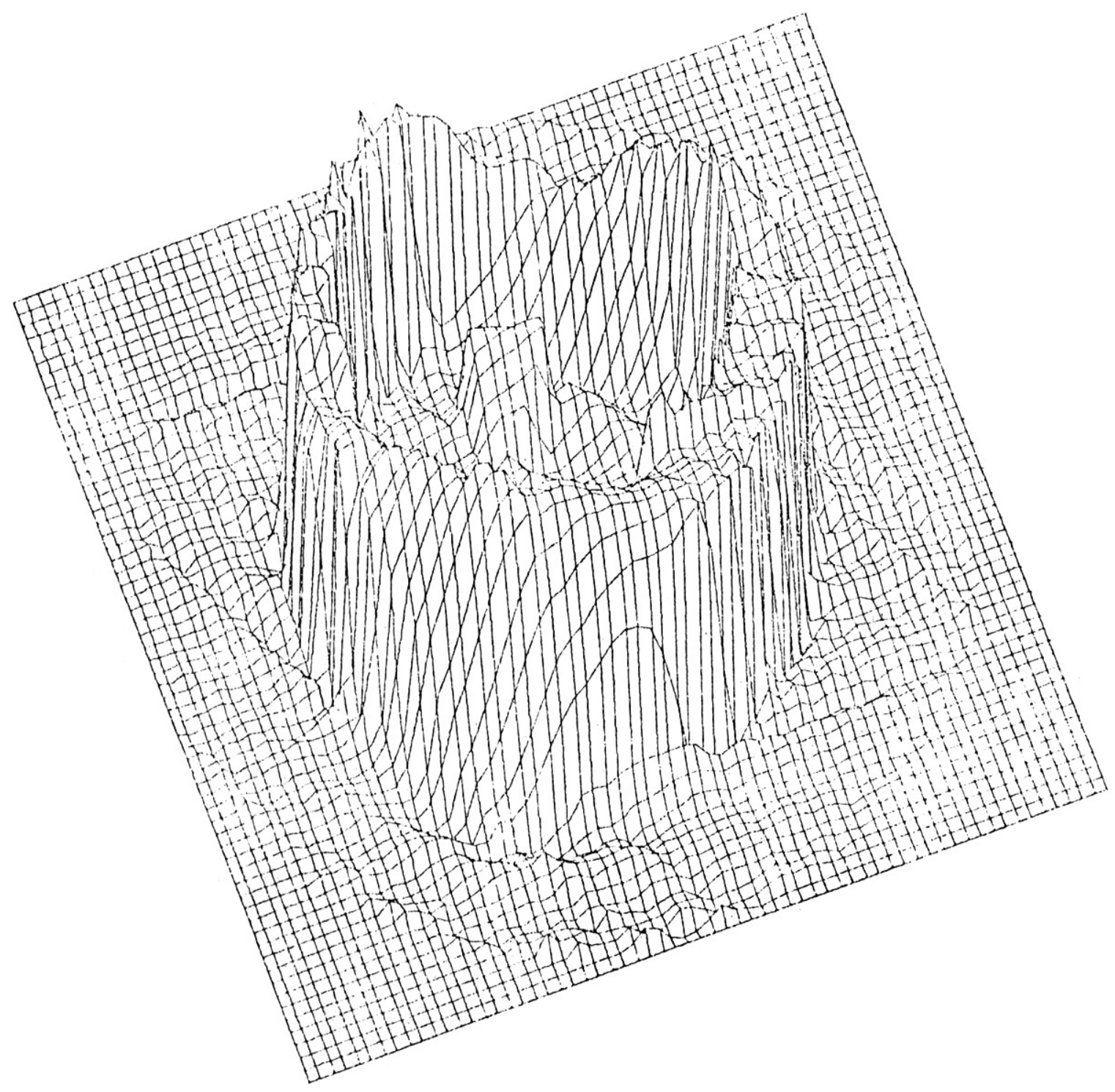




\section{Figure 8}

Reconstruction of the second test object from 60 projections in $[0, \pi[$

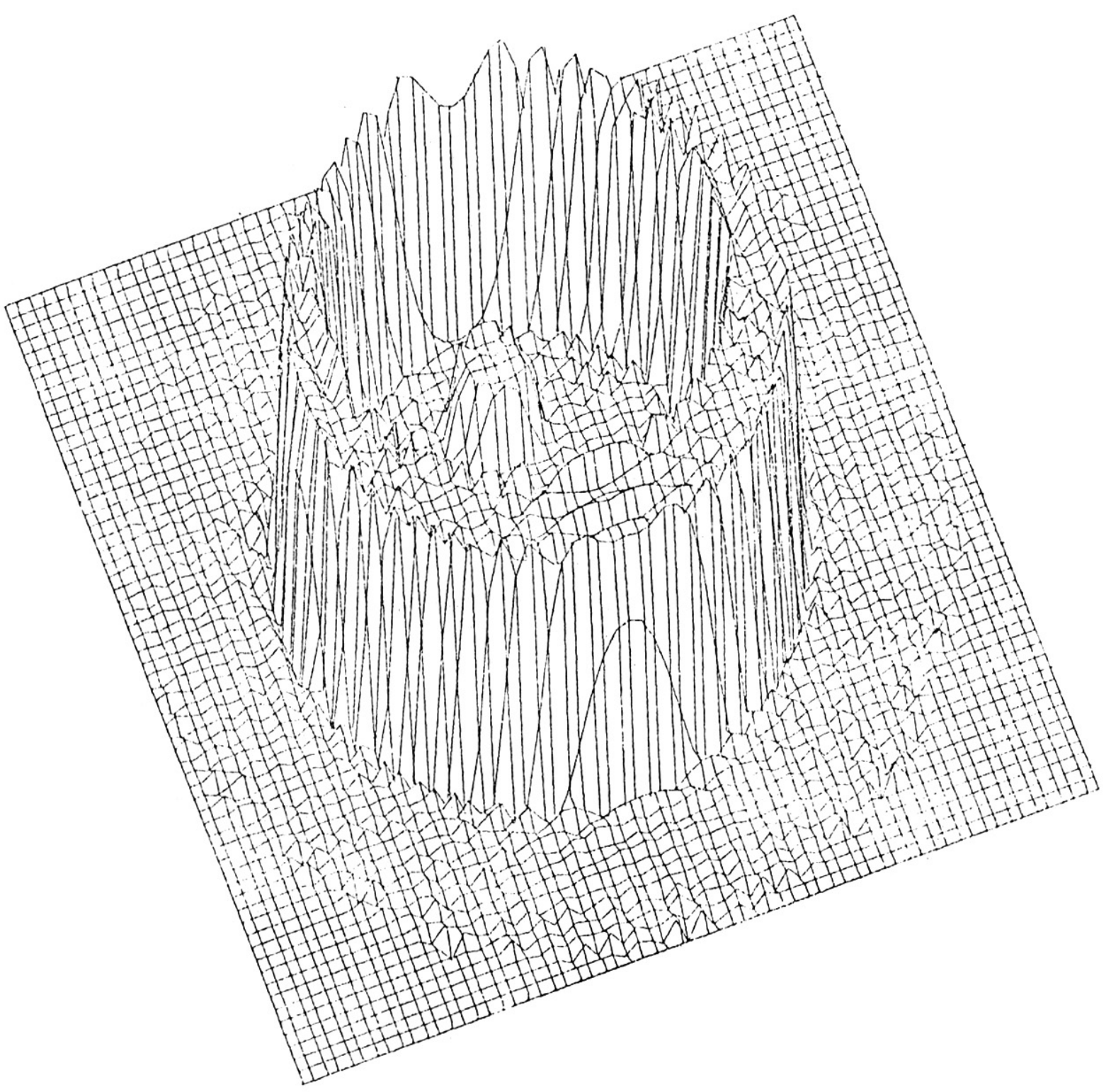


Figure 9

Cut at $y=0$

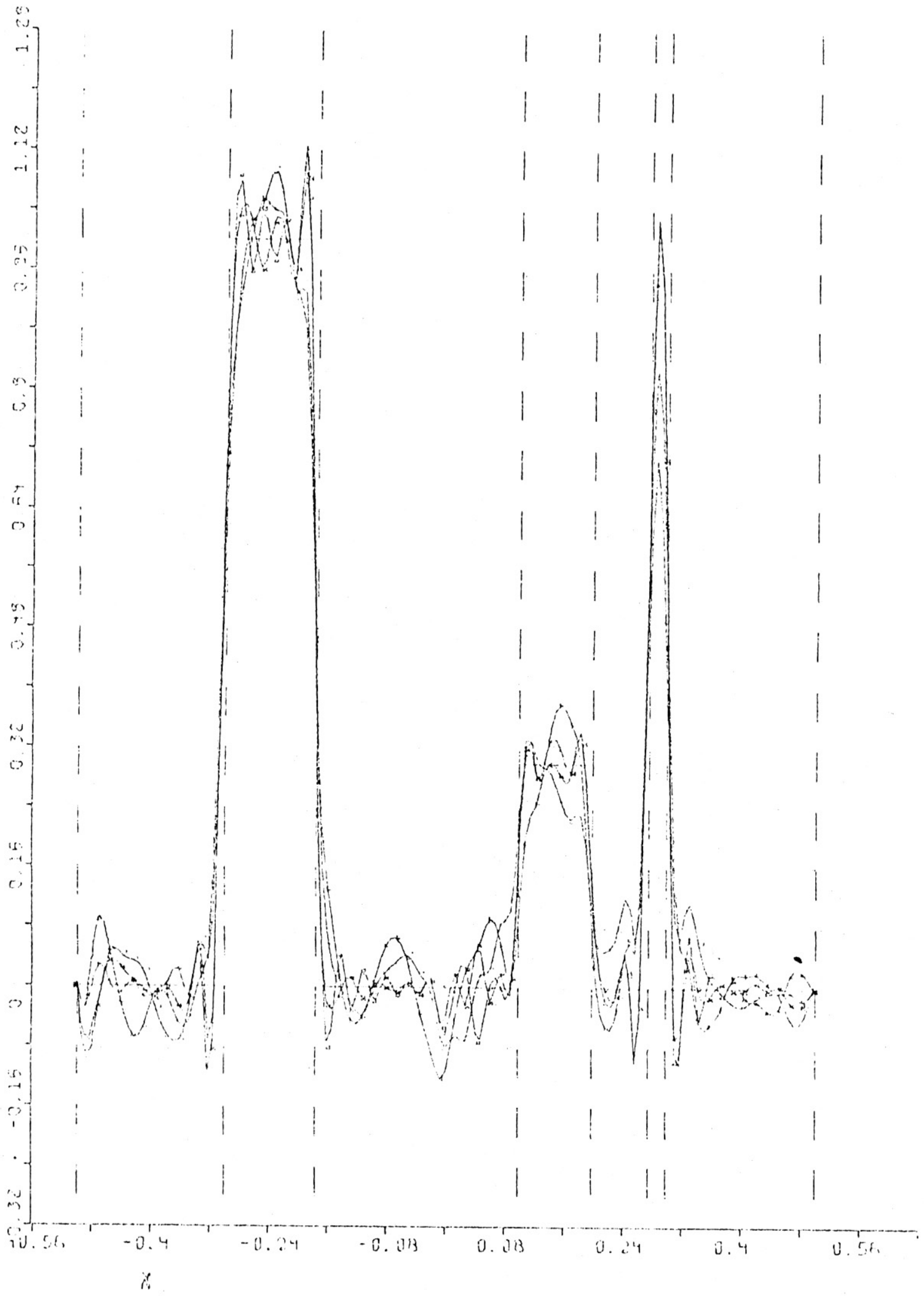




\section{References}

[1] BUDINGER, T.F. - GULBERG, G.T.: Three-dimensional recon struction in nuclesr medicine emission imaging. IEEE Transactions on Nuclear Science 21, 2-20, 1974

[2] GORDON, R. - HERMAN, G.T.: Reconstruction of pictures from their projections. Comm. ACM 14, 759-768, 1971

[3] LAX, P.D. - PHILLIPS, R.S.: The Paley-Wiener theorem for the Radon transform. Comm. Pure Appl. Math. 23, 409-424, 1970

Correction : ibid 24, 279, 1971

[4] LIONS, J.L. - MAGENES, E.: Problèmes aux limites non homo gènes, Vol. 1, Paris : Dunod, 1968

[5] LUDWIG, D.: The Radon transform on Euclidean space, Comm. Pure Appl. Math. 19, 49-81, 1966

[6] NATTERER, F.: A Sobolev space analysis of picture recon struction, to appear in SIAM J. Appl. Math.

[7] NATTERER, F.: Genauigkeitsfragen bei der numerischen Rekonstruktion von Bildern to appear in Basel : ISNM Birckhäuser Verlag

[8] ODEN, J.T. - REDDY, J.N.: An introduction to the mathematical theory of finite elements, New York : John Wiley\&Sons, 1976

[9] RAMACHANDRAN, G.N. - LAKSHMINARAYANAN, A.V.: Three - dimen sional reconstruction from radiographs and electron micro graphs : application of convolutions instead of Fourier transform.

Proc. Nat. Acad. Sci. U.S. 68, 2236-2240, 1971

[10] SMITH, K.T. - SOLMON, D.C. - WAGNER, S.L.: Practical and mathematical aspects of the problem of reconstructing objects from radiographs. Bul. AMS 83, 1227-1270, 1977 
[11] TRIEBEL, H.: Interpolation theory, function spaces, differential operators, Amsterdam : North Holland, 1978

[12] WAGNER, W.: Incomplete scan geometries in fast x-ray scanners. to appear

Dr. Alfred Louis

Fachbereich 10 -

Angewandte Mathematik und Informatik

der Universität des Saarlandes

D-6600 Saarbrücken

BRD 